\title{
THE GENERAL CATALOGUE OF STELLAR PROPER MOTION WITH RESPECT TO GALAXIES WITH ASTROPHYSIC SUPPLEMENT
}

\author{
N.V. KHARCHENKO \\ Main Astronomical Observatory \\ Ukrainian Academy of Sciences \\ 252127 Kiev \\ USSR
}

\begin{abstract}
Within the limits of the programme of studying of the Main Meridional Section of the Galaxy the General Catalogue of astrometric data of 26500 stars has been compiled. Corrections to the precession constants and stellar secular parallaxes have been determined.
\end{abstract}

The programme of studying of the Main Meridional Section of the Galaxy (MEGA) (Einasto et al., 1985) provides for the creation of catalogues of proper motions with respect to galaxies, equatorial coordinates, stellar magnitudes in the UBVR system, absolute stellar magnitudes, effective temperatures, metal contents of all stars down to $12^{\mathrm{m}}$ in 47 areas of the sky, each of size $5^{\circ} \times 5^{\circ}$, and astrometric and photometric data only for stars down to $15^{\mathrm{m}}-16^{\mathrm{m}}$ in areas whose diameters are on the order of $1^{\circ} 20^{\prime}$.

The General Catalogue of astrometric data was compiled. Next basic principles were used for the compilation of the Catalogue: the application of original observations and all of bibliographic data; the analysis of systematic and accidental errors of stellar characteristics; the determination of optimal level of taking the average of these errors; the reduction of various stellar characteristics to their systems; the choice of priorities for further using of data, if these data were determined by means of various methods or with various errors.

The observations were carried using the long-focus double astrograph in Kiev. In order to improve the precision of proper motions, reference stars were selected as kinematically homogeneous group of distant stars. For the consideration of the magnitude equation in proper motions a statistical technique using the composed catalogue data only (Kharchenko, 1984) has been developed. Proper motions in reference to galaxies were converted to the General Catalogue system. This one was obtained from comparison of absolute proper motions from catalogues received on the Faint Stars Plan and AGK3. The General Catalogue contains 26500 stars.

The maximum of the distribution of r.m.s.errors of absolute proper motions is 0".6 per century. Equatorial coordinates were calculated with respect to positions of AGK3 and SAO stars with r.m.s. errors of $\pm(0.3-0.6)$. Astrophysical data of some stars were found in bibliographic sources.

Using the General Catalogue and the Pulkovo Catalogue, which was reduced to the system of General Catalogue in the sense of the magnitude equation consideration, corrections to the precession constant were determined: $\Delta k=-0.24 \pm 0.17$ and $-0.03 \pm 0.14 ; \Delta n=+0.24 \pm 0.16$ and $+0.29 \pm 0.14$ arcsec/century respectively. 
Table 1. Stellar characteristics of GC

\begin{tabular}{llll}
\hline $\begin{array}{l}\text { Stellar } \\
\text { characteristic }\end{array}$ & $\begin{array}{l}\text { Number } \\
\text { of stars }\end{array}$ & \multicolumn{2}{l}{$\begin{array}{l}\text { Limiting stellar magnitude B } \\
\text { (completeness until B) }\end{array}$} \\
\hline No. BD & 12950 & $12 \mathrm{~m} .5$ & $\left(10^{\mathrm{m}}\right)$ \\
RA, DEC, PM & 26436 & 16 & $(13.5)$ \\
B photographic & 23495 & 16 & $(13.5)$ \\
V photovisual & 7777 & 11 & $(10)$ \\
U photoelectric & 1339 & 11 & \\
B photoelectric & 1719 & 11 & \\
V photoelectric & 1720 & 11 & \\
Spectral class & 12649 & 12.5 & $(10)$ \\
Luminocity class & 1405 & 11 & \\
Radial velocity & 1266 & 11 & \\
\hline
\end{tabular}

On the basis of absolute proper motions, taken from the General Catalogue, AGK3 and papers of Deutsch (1947), and Fatchikhin (1970), stellar secular parallaxes were determined on high, middle and low galactic latitudes.

Table 2. Secular parallaxes $(\mu<5$ "/century )

\begin{tabular}{|c|c|c|c|c|c|c|c|}
\hline $\mathbf{B}=$ & 9.5 & $10^{m} 5$ & $\frac{11^{\mathrm{m} 5}}{(h / \rho)}$ & $\begin{array}{c}12 \mathrm{~m} .5 \\
0: 0001\end{array}$ & $13 \mathrm{~m} .5$ & 14.5 & $15^{\mathrm{m}} \cdot 5$ \\
\hline$+75^{\circ}$ & 180 & 160 & 145 & 130 & 112 & 100 & 95 \\
\hline$+45^{\circ}$ & 142 & 122 & 110 & 98 & 87 & 80 & 78 \\
\hline$+20^{\circ}$ to $-20^{\circ}$ & 160 & 135 & 116 & 100 & 83 & 72 & 60 \\
\hline
\end{tabular}

\section{References}

Deutsch, A.N. (1947), Izv. Glavnoj astron. observ. v Pulkovo, 17, No. 36, pp. 2-59.

Einasto, J., Maluto, V.D., Kharchenko, N.V. (1985), Astron.circular, No. 1394, pp. 1-6.

Fatchikhin, N.V. (1970), Astron. Zhurn., 47, 619-632.

Kharchenko, N.V. (1984), Astrometrija i astrofizika, 52, 3-8.

\section{Discussion}

MURRAY: Do your values of secular parallax in different latitudes depend on different directions to the solar apex?

KHARCHENKo: We had determined the secular parallaxes by means of the equation of Kovalsky-

Ery. Then the direction to the solar apex was obtained together with secular parallaxes and this direction varies for different galactic latitudes.

BROSCHE: What is the typical epoch difference on which your proper motions are based?

KHARCHENKO: The mean epoch difference of pairs of plates is 24.6 years. 